\title{
REPLACING HOMOTOPY ACTIONS BY TOPOLOGICAL ACTIONS. II
}

\author{
LARRY SMITH
}

\begin{abstract}
A homotopy action of a group $G$ on a space $X$ is a homomorphism from $G$ to the group $\operatorname{HAUT}(X)$ of homotopy classes of homotopy equivalences of $X$. George Cooke developed an obstruction theory to determine if a homotopy action is equivalent up to homotopy to a topological action. The question studied in this paper is: Given a diagram of spaces with homotopy actions of $G$ and maps between them that are equivariant up to homotopy, when can the diagram be replaced by a homotopy equivalent diagram of $G$-spaces and $G$-equivariant maps? We find that the obstruction theory of Cooke has a natural extension to this context.
\end{abstract}

\section{INTRODUCTION}

A homotopy action of a group $G$ on a space $X$ is a homomorphism

$$
\rho: G \rightarrow \operatorname{HAUT}(X),
$$

where $\operatorname{HAUT}(X)$ is the group of homotopy classes of homotopy equivalences of $X$. (We abbreviate homotopy action to ho-action.) If $G$ acts on the space in the usual sense then the induced homotopy action of $G$ on $X$ is called topological. A homotopy action $\rho^{\prime}$ of $G$ on $X^{\prime}$ is equivalent to a homotopy action $\rho^{\prime \prime}$ on $X^{\prime \prime}$ iff there exists a homotopy equivalence $f: X^{\prime} \rightarrow X^{\prime \prime}$ such that

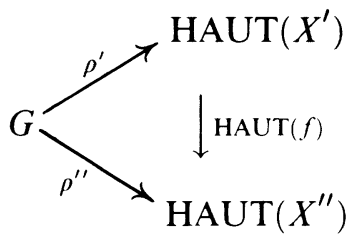

is commutative, where $\operatorname{HAUT}(f)$ is defined by $[g] \rightarrow\left[f g f^{\wedge}\right]$, where $f^{\wedge}$ is a homotopy inverse of $f^{\wedge}$.

In [3] George Cooke studied the problem of when a homotopy action is equivalent to a topological action. The present paper is a direct generalization of Cooke's ideas and methods, and extends Cooke's work to diagrams of spaces with a homotopy action and maps between them that are equivariant up to

Received by the editors April 20, 1988.

1980 Mathematics Subject Classification (1985 Revision). Primary 55P99; Secondary 55R05, $55 \mathrm{~S} 37$. 
homotopy. For spaces $X^{\prime}$ and $X^{\prime \prime}$ with ho-actions of $G$, a map $f: X^{\prime} \rightarrow X^{\prime \prime}$ is called homotopy equivariant (abbreviated ho-equivariant) iff for $g \in G$, the diagram

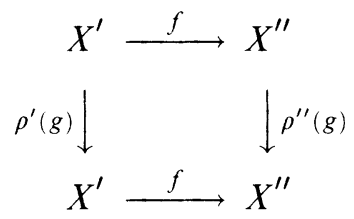

is homotopy commutative, where $\rho^{\prime}(g)$, resp. $\rho^{\prime \prime}(g)$, is a map in the homotopy class $\rho^{\prime}(g)$, resp. $\rho^{\prime \prime}(g)$. Put another way, if TOP denotes the category of spaces and maps, and ho-TOP the category of spaces and homotopy classes of maps, then a ho-action of $G$ on $X$ is just a $G$-object in ho-TOP, and $f$ is ho-equivariant iff it is a morphism in ho-TOP of $G$-objects. A natural question to ask is: When is a homotopy equivariant map equivalent to a $G$-map, in the sense that there are homotopy equivalences

$$
\phi^{\prime}: X^{\prime} \rightarrow Y^{\prime}, \quad \phi^{\prime \prime}: X^{\prime \prime} \rightarrow Y^{\prime \prime},
$$

topological actions of $G$ on $Y^{\prime}$ and $Y^{\prime \prime}$, and a $G$-equivariant map $h: Y^{\prime} \rightarrow Y^{\prime \prime}$ such that the diagram

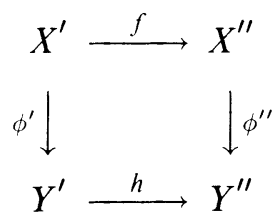

is homotopy commutative? More generally one can ask for a topological realization of a diagram of spaces with $G$-ho-action where the maps are ho-equivariant. The purpose of this paper is to provide a homotopy theoretic treatment of this problem extending the work of Cooke [3]. This is done in $\S 3$. To do so however requires an analogous extension of the work of Allaud [1] on classifying spaces for Hurewicz fibrations. This is accomplished in $\S 2$. In $\S 4$ we discuss some applications, and $\S 5$ is an attempt to relate our work to that of Dwyer and Kan $[4,5,6]$.

I want to thank Chariya Peterson for helpful discussions on the material of $\S 2$, and the University of Virginia for providing me with a working environment.

\section{A CLASSIFICATION THEOREM FOR DIAGRAMS OF FIBRATIONS}

By a homotopy commutative diagram we understand a functor $X: D \rightarrow$ ho-TOP where $D$ is a kittygory (:= small category). If $B$ is a fixed space we denote by TOP / $B$ the category with objects $\gamma: X \rightarrow B, \rho \in \operatorname{Morph}($ TOP), and morphisms the strictly commutative triangles (in TOP):

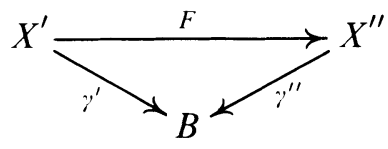


Homotopies of morphisms are fibrewise homotopies, and the associated category with the same objects and fibrewise homotopy classes of maps will be denoted by ho-TOP $/ B$. A homotopy commutative diagram of Hurewicz fibrations over the pointed space $B$ consists of:

- a functor $E: D: \rightarrow$ ho-TOP $/ B$ where $d \in \operatorname{Obj}(D), \gamma(d): E(d) \rightarrow B$ is a Hurewicz fibration, and

- a natural transformation $\nu: X \rightarrow o(E)$, where $o:$ ho-TOP $/ B \rightarrow$ ho-TOP is the forgetful functor, such that for every $d \in \operatorname{Obj}(D), \nu(d): X(d) \rightarrow$ $E(d)$ is a homotopy equivalence onto the fibre $F(*)=\gamma(d)^{-1}(*), * \in B$ the basepoint.

Example. If $D$ is the category with two objects $\{0,1\}$ and one morphism $0 \rightarrow 1$ then a homotopy ccmmutative diagram of Hurewicz fibrations of type $D$ consists of two Hurewicz fibrations $E(0) \downarrow B, E(1) \downarrow B$ and a homotopy class of fibrewise maps

$$
E(0) \rightarrow E(1) \downarrow B
$$

together with homotopy equivalences $X(i)=\pi(i)^{-1}(*)$ for spaces $X(i), i=$ 0,1 .

For a fixed kittygory $D$ and pointed space $B$ we denote (following [1]) by $H_{D}(B, X)$ the set of equivalence classes of homotopy commutative diagrams of Hurewicz fibre spaces over $B$ where the fibres are of type $X: D \rightarrow$ ho-TOP. For a fixed functor $X: D \rightarrow$ ho-TOP,$H_{D}(-, X)$ defines a cofunctor $\mathrm{TOP}_{*} \rightarrow$ ENS, where ENS is the category of sets.

Theorem 2.1. Let $D$ be a kittygory and $X: D \rightarrow$ ho-TOP a $C W$-valued functor. Then the functor:

$$
H_{D}(-, X): \mathrm{TOP}_{*} \rightarrow \text { ENS }
$$

is a homotopy functor (in the sense of $E$. H. Brown).

Proof. Introduce the category

$$
\text { FUN }:=\mathrm{FUN}\left(D, \text { ho-TOP } / \mathrm{TOP}_{*}\right)
$$

of functors from $D$ to the homotopy category of spaces over pointed spaces. This is a closed model category (see e.g. [2]) in a natural way. Note that a homotopy commutative diagram of Hurewicz fibrations of type $D$ and fibres of type $X: D \rightarrow$ ho-TOP is a fibration (= fibrant object) in FUN. The argument of Allaud [1, III] being "formal homotopy theory" goes through in this context to yield the desired conclusion.

Corollary 2.2. Let $D$ be a kittygory and $X: D \rightarrow$ ho-TOP a $C W$-valued functor. Then there exists a pointed space $B_{X}$, and a homotopy commutative diagram $U: D \rightarrow$ ho-TOP $/ B_{X}$ of Hurewicz fibrations, such that the natural transformation

$$
\left[-, B_{X}\right] \rightarrow H_{D}(-, X)
$$

given by pullback is an equivalence of functors. 
Proof. This follows from Brown's theorem [7].

The space $B_{X}$ has an alternate description that will be of use in the next section. We turn to this now.

Let $X: D \rightarrow$ ho-TOP be a homotopy commutative diagram. Denote by $H E(X)$ the space of all self-homotopy equivalences of $X$, i.e., an element of $H E(X)$ is an assignment to each $d \in \operatorname{Obj}(D)$ a self-homotopy equivalence $\Theta(d): X(d) \rightarrow X(d)$ such that upon passing to homotopy classes $\Theta$ defines a natural equivalence of the functor $X$ with itself. Note that $H E(X)$ is an associative $H$-space with unit, and $\pi_{0}(H E(X)) \simeq \operatorname{HAUT}(X)$ is the group of self-equivalences of the functor $X$. Since $H E(X)$ is associative it has a classifying space $B H E(X)$. Over $B H E(X)$ there is a homotopy commutative diagram of principal fibrations where for each $d \in \operatorname{Obj}(D)$ the fibre $(=H$ space of the bundle) is $H E(X(d))$, the $H$-space of self-homotopy equivalences of $X(d)$. Passing to the associated fibrations with fibre $X(d)$ we obtain a functor $V: D \rightarrow$ ho-TOP/BHE $(X)$ which has a natural structure of a homotopy commutative diagram of Hurewicz fibrations over $B H E(X)$ with fibres of type $X$. Hence we obtain a classifying map

$$
v: B H E(X) \rightarrow B_{X}
$$

for $V: D \rightarrow$ ho-TOP $(B H E(X))$ from (2.2). The following is then clear (see e.g. [1]).

Theorem 2.3. Let $D$ be a kittygory and $X: D \rightarrow$ ho-TOP a $C W$-valued functor. Then $v: B H E(X) \rightarrow B_{X}$ is a homotopy equivalence.

\section{HOMOTOPY ACTIONS ON HOMOTOPY COMMUTATIVE DIAGRAMS}

Let $D$ be a kittygory and $X: D \rightarrow$ ho-TOP a homotopy commutative diagram. By a homotopy action of the group $G$ on $X$ we understand a homomorphism $\rho: G \rightarrow \operatorname{HAUT}(X)$ where $\operatorname{HAUT}(X)$ is the group of automorphisms of the functor $X$. If $X^{\prime}, X^{\prime \prime}: D \rightarrow$ ho-TOP and $\rho^{\prime}, \rho^{\prime \prime}$ are ho-actions of $G$ on $X^{\prime}, X^{\prime \prime}$, then a natural transformation $F: X^{\prime} \rightarrow X^{\prime \prime}$ is an equivalence iff the diagram

commutes.

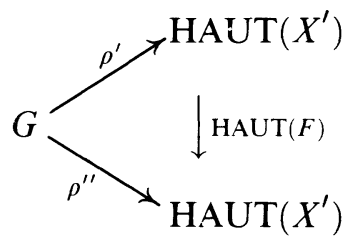

For a homotopy commutative diagram $X: D \rightarrow$ ho-TOP, an action of $G$ on $X$ consists of a homomorphism $d \in \operatorname{Obj}(D)$ such that

$$
\rho(d): G \rightarrow H E(X(d))
$$

and a $G$-equivariant map $\phi \in \operatorname{Morph}(D)$ such that

$$
f(\phi): X(\text { domain }(\phi)) \rightarrow X(\operatorname{range}(\phi)) ;
$$


passing to homotopy classes gives a homotopy action of $G$ on $X$.

Such an action is called topologically realizable, or simply topological. (N.B. The group $G$ actually acts on $X$, the maps of the diagram are $G$-maps, but the diagram commutes only up to homotopy. See $\S 5$ for a further discussion of this point.)

For a diagram $X: D \rightarrow$ ho-TOP we denote by $H E(X)$ the associative $H$ space of all tuples $h(d) \in H E(X(d))$ such that passing to homotopy classes gives an automorphism of the functor $X$. The passage to homotopy classes defines an $H$-map

$$
H E(X) \rightarrow \operatorname{HAUT}(X)=\pi_{0}(H E(X))
$$

and induces a fibration

$$
\pi: B H E(X) \rightarrow B \text { HAUT }(X)
$$

whose fibre is $B H E_{\mathrm{id}}(X)$, where $H E_{\mathrm{id}}(X)$ is the sub- $H$-space of tuples $h(d)$, such that $h(d)$ is homotopic to $\operatorname{id}_{X(d)}$.

Theorem 3.1. Let $D$ be a kittygory, $X: D \rightarrow$ ho-TOP a homotopy commutative diagram and $\rho: G \rightarrow \operatorname{HAUT}(X)$ a ho-action of $G$ on $X$. Then $\rho$ is topologically realizable iff the lifting problem

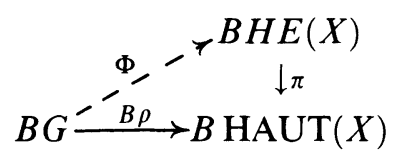

has a solution.

Proof (after Cooke). Suppose that $\rho$ is topologically realizable by $\mu: G \rightarrow$ $H E(Y)$. Set $F: X \rightarrow Y$ be an equivalence. WLOG we may suppose that $G$ acts freely on each $Y(d)$ for $d \in \operatorname{Obj}(D)$. We then have principal $G$-bundles

$$
Y(d) \rightarrow Y(d) / G, \quad d \in \operatorname{Obj}(D) .
$$

Let these be classified by

$$
\phi(d): Y(d) \rightarrow B G .
$$

Since the maps in the diagram $Y$ are equivariant, we have induced maps

$$
f / G: Y(\operatorname{domain}(f)) / G \rightarrow Y(\operatorname{range}(f)) / G
$$

and a functor

$$
Y / G: D \rightarrow \text { ho-TOP. }
$$

Convert each of the maps $\phi(d)$ into a fibration (see [2]). The fibre of $\phi(d)$ is then homotopy equivalent to $Y(d)$, which via $F(d)$ is homotopy equivalent to $X(d)$. Thus we have described a functor

$$
Y / G: D \rightarrow \text { ho-TOP } / B G
$$

which is a homotopy commutative diagram of Hurewicz fibrations of type $X: D \rightarrow$ ho-TOP. By (2.1) and (2.2) we therefore obtain a classifying map

$$
\Theta: B G \rightarrow B H E(X)
$$


for $Y / G$. To check that the diagram

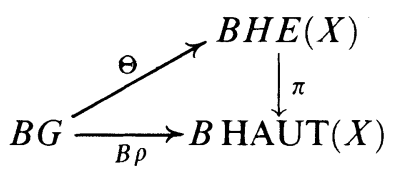

homotopy commutes it suffices to check commutativity on $\pi_{1}$, where it is clear.

Conversely given a solution to the lifting problem $(*)$ we obtain an induced diagram of Hurewicz fibrations

$$
W: D \rightarrow \text { ho-TOP } / B G .
$$

Let $E G \downarrow^{u} B G$ be the universal covering and form the pullback functor

$$
u^{*} W: D \rightarrow \text { ho-TOP } / E G \text {. }
$$

Note that $G$ acts on each $u^{*} W(d)$ and each map $u^{*} W(\phi)$ is $G$-equivariant. Moreover, $E G$ being contractible, $u^{*} W$ is equivalent to $\left.u^{*} W\right|_{*}$ (= restrict $u^{*} W$ to a point) which is just $X$. Thus $u^{*} W$ provides a topological realization of $X$.

Thus as in Cooke [3] the realization problem is reduced to an obstruction theory problem. Specifically:

Corollary 3.2. Let $D$ be a kittygory and $\rho: G \rightarrow \operatorname{HAUT}(X)$ a ho-action of $G$ on the homotopy commutative diagram $X: D \rightarrow$ ho-TOP. Then $\rho$ is realizable topologically iff a sequence of obstructions

$$
\Theta^{n}[\rho] \in H^{n}\left(G ; \pi_{n-2}\left(H E_{\mathrm{id}}(X)\right)\right) \mid n \geq 3
$$

all vanish.

Proof. This follows by noting that

$$
\pi_{1}(B H E(X)) \rightarrow \pi_{1}(B \operatorname{HAUT}(X))
$$

is an isomorphism, and that $B H E_{\mathrm{id}}(X)$ is the fibre of $\pi$.

\section{Applications}

The main results of the previous section show that a homotopy action of $G$ on a homotopy commutative diagram $X: D \rightarrow$ ho-TOP is equivalent to a topological action iff a sequence of obstructions

$$
\Theta^{n} \in H^{n}\left(G ; \pi_{n-2}\left(H E_{\text {id }}(X)\right)\right) \mid n \geq 3
$$

all vanish. Note that $H E_{\text {id }}(X)$ is simply the component of the identity map in $\operatorname{map}(X, X)$. If $X$ is $p$-complete for a prime $p$ in the sense that $X(d)$ is $p$-complete for every $d \in \operatorname{Obj}(D)$, then the $\operatorname{space} \operatorname{map}(X, X)$ [2] is also $p$ complete and hence a fortiori the component of the identity. This leads to the following result. 
Proposition 4.1. Let $G$ be a finite group of order prime to $p, p$ a prime, and $X: D \rightarrow$ ho-TOP a p-complete homotopy commutative diagram. Then any ho-action of $G$ on $X$ is topologically realizable.

Proof. Since $X$ is $p$-complete so is $H E_{\mathrm{id}}(X)$. Therefore $\pi_{m}\left(H E_{\mathrm{id}}(X)\right)$ is $p$ complete. Since $p|| G \mid$ it follows that the groups $H^{n}\left(G ; \pi_{n-2}\left(H E_{\text {id }}(X)\right)\right)$ all vanish.

The groups $G=Z$ and $G=Z \times Z$ are special in that $B G=S^{1}$ or $S^{1} \times S^{1}$ have dimension at most 2 . Since the first obstruction to realizing a ho-action by a topological action occurs in dimension 3 we obtain:

Proposition 4.2. Let $X: D \rightarrow$ ho-TOP be a homotopy commutative diagram. Then any ho-action of $Z$ or $Z \times Z$ can be realized topologically.

In particular this says that a self-homotopy equivalence $f: X \rightarrow X$ of a space $X$ is realizable up to homotopy by a homeomorphism $h: Y \rightarrow Y$ of a homotopy equivalent space.

Finally we note the analog of $[3,2.4]$ for maps.

Proposition 4.3. Let $R$ be a commutative ring and $G$ a finite group whose order is invertible in $R$. If $\rho$ is a ho-action of $G$ on a homotopy commutative diagram $X: D \rightarrow$ ho-TOP then there exists a homotopy commutative diagram $X / G: D \rightarrow$ ho-TOP and a morphism $\pi: X \rightarrow X / G$ such that for all $d \in \operatorname{Obj}(D)$

$$
\pi(d): H^{*}(X(d) ; R) \rightarrow H^{*}(X(d) ; R)^{G} .
$$

Proof. The homotopy action $\rho$ induces a homotopy action on the $R$ completion $X_{R}: D \rightarrow$ ho-TOP of $X$. By (4.1) $\rho_{R}$ is topologically realizable. Let

$$
\phi: X \rightarrow Y: D \rightarrow \text { ho-TOP }
$$

to be a topological realization of $\rho$ on $Y$. WLOG we may suppose $G$ acts freely on $Y$. A standard transfer argument now shows the composite $X \rightarrow$ $Y \rightarrow Y / G$, where $Y / G$ is the diagram of orbit spaces, satisfies the conclusion.

\section{Closing Remarks}

Suppose that $D$ is a kittygory and that the group $G$ acts on the homotopy commutative diagram $X: D \rightarrow$ ho-TOP. A natural question to ask is: Does there exist a strictly commutative diagram $Y: D \rightarrow$ TOP, an action of $G$ on $Y$ (so all spaces $Y(d)$ are $G$-spaces and all maps $Y(\phi)$ are $G$-equivariant) and a homotopy equivalence $X \rightarrow$ ho- $Y$, where ho- $Y$ is the composite $Y: D \rightarrow$ TOP $\rightarrow$ ho-TOP ? One method to approach this problem would be to bring in the work of Dwyer and Kan [4, 5, 6]. These authors study the question: Given a homotopy commutative diagram $X: D \rightarrow$ ho-TOP, when does there exist a commutative diagram $Y: D \rightarrow$ TOP and a homotopy equivalence $X \rightarrow$ ho $Y$ ? They prove [5] there is an obstruction theory for this problem analogous to that 
of Cooke [3]. In particular they state that when $D=G$ (where $G$ denotes the category with one object and endomorphism set $G$ ), their obstruction theory reduces to that of Cooke. It would be interesting to see the relations between all these results spelled out in detail.

N.B. If $D$ contains no closed loops (e.g. $D$ is a tree) then there is no distinction between homotopy commutativity and strict commutativity. This suggests that perhaps the fundamental group of the components of the topological realization of the category $D$ should enter in some way. The higher homotopy groups enter into the situation when $D$ is endowed with commuting homotopies etc.

\section{REFERENCES}

1. G. Allaud, On the classification of fibre spaces, Math. Z. 92 (1966), 110-125.

2. A. K. Bousfield and D. M. Kan, Homotopy limits, completions and localizations, Lecture Notes in Math., vol. 304, Springer-Verlag, 1972.

3. G. E. Cooke, Realizing homotopy actions by topological actions, Trans. Amer. Math. Soc. 237 (1978), 391-406.

4. W. G. Dwyer and D. M. Kan, Function complexes for diagrams of simplicial sets, Indag. Math. 45 (1983), 139-147.

5. __ Realizing diagrams in the homotopy category by means of diagrams of simplicial sets.

6. __ A classification theorem for diagrams of simplicial sets, Topology 23 (1984), 139-153.

7. E. H. Spanier, Topology, McGraw-Hill, 1966.

Mathematisches Institut der Georg August Universität, Bunsenstrasse 3/5, D-3400 GötTINGEN, West Germany 